\title{
Business Processes Modeling Recommender Systems: User Expectations and Empirical Evidence
}

\author{
Michael Fellmann ${ }^{1 *}$, Novica Zarvić $^{2}$, and Oliver Thomas ${ }^{2}$ \\ ${ }^{1}$ Institute of Computer Science, University of Rostock, Rostock, Germany \\ 2 Information Management and Information Systems Group, Osnabrück University, \\ Osnabrück, Germany \\ michael.fellmann@uni-rostock.de, \\ \{novica.zarvic, oliver.thomas\}@uni-osnabrueck.de
}

\begin{abstract}
Recommender systems are in widespread use in many areas, especially electronic commerce solutions. In this contribution, we apply recommender functionalities to business process modeling and investigate their potential for supporting process modeling. To do so, we have implemented two prototypes, demonstrated them at a major fair and collected user feedback. After analysis of the feedback, we have confronted the findings with the results of the experiment. Our results indicate that fairgoers expect increased modeling speed as the key advantage and completeness of models as the most unlikely advantage. This stands in contrast to an initial experiment revealing that modelers, in fact, increase the completeness of their models when adequate knowledge is presented while time consumption is not necessarily reduced. We explain possible causes of this mismatch and finally hypothesize on two "sweet spots" of process modeling recommender systems.
\end{abstract}

Keywords: Recommender systems, Semantic modeling, Process-Oriented information system, Empirical evaluation, Experiment.

\section{Introduction}

Recommender systems are generally characterized by the fact that they "generate meaningful recommendations to a collection of users" [1]. Such recommender functionalities are features that simplify and ease the work and interaction of a user with the system under consideration. They are "providing suggestions for items to be of use" and for "supporting their users in various decision-making processes" [2]. This is a very relevant issue in today's world and information society, because humans are often overwhelmed with the increasing amount of available information. Recommender functionality is, for instance, a well-known feature of search engines

\footnotetext{
* Corresponding author

(C) 2018 Michael Fellmann et al. This is an Open Access article licensed under the Creative Commons Attribution License (CC BY 4.0), https://creativecommons.org/licenses/by/4.0
}

Reference: M. Fellmann, N. Zarvić, and O. Thomas, "Business Processes Modeling Recommender Systems: User Expectations and Empirical Evidence," Complex Systems Informatics and Modeling Quarterly, CSIMQ, no. 14, pp. 64-79, 2018. Available: https://doi.org/10.7250/csimq.2018-14.05

Additional information. Author's ORCID iD: M. Fellmann - orcid.org/0000-0003-0593-4956. PII S225599221800084X. Received: 14 February 2018. Accepted: 9 April 2018. Available online: 30 April 2018. 
and/or market places on the internet (e.g. amazon.com), where online users are supported in dealing with the existing information overload.

However, recommender functionality is not bound to internet usage and electronic commerce. It is also capable to support users in other fields of activities, for instance in the context of Business Process Management (BPM) being also a relevant market [3]. Organizations can benefit from using tools that are supporting the users, or more specifically the modelers of business processes. Such a support might help to more conveniently create models conforming to specified naming conventions and sticking to well-defined abstraction levels [4]. Regarding the former, terminological problems are amongst the main challenges when using conceptual (process) models [5]. Regarding the latter, recommender systems might help to know where to start and where to stop modeling and on which abstraction level to model [6], [7].

In this article we present a recommendation-based business process modeling approach, which is supported by two prototypical tools. The SEMPHIS (Semantic Modeling of Process-oriented Information Systems) tools put recommender functionalities for modeling business processes into execution. More specifically, we are investigating the usefulness of our solution on the basis of a preliminary feedback from potential users' which is based on a recent survey. We then contrast this feedback with the results from an experiment. Hence, the goal of this article is to get the first empirical evidence, based on both, the estimation and the experiment, of how well process modeling recommender systems might be able to ease daily work of modelers in the BPM domain. This article is an extended and revised version of [8].

Our article is structured as follows. Section 2 provides some important background information on recommender systems in general and on our prototypical solution in particular. Then, Section 3 briefly describes the research process followed, before we present our results from fairgoers in Section 4; and from an experiment in Section 5. In Section 6, the article is concluded and further research activities are outlined.

\section{Background Information}

\subsection{On Related Research Directions}

The fundamental idea of our approach is to support the modeler with a recommendation feature that leverages existing process knowledge. Therefore, research regarding modeling support using knowledge representations, in a broad sense, is related to our work. Such research can be found in several areas.

Reference-based model construction. The construction of models by leveraging the existing models is addressed in the area of reference modeling. New models are constructed by adapting existing models which may be selected from a reference model catalogue [9], a repository of process fragments [10], a community portal [11] or which may be included in a modeling tool (e.g. IT Infrastructure Library is included in several modeling tools) [12]. Although model reuse is intended, recommendation features have not yet gained much attention in this research direction.

Reuse-based model construction. Reuse is recently discussed in BPM as a more holistic approach in comparison to reference modeling in the sense of spanning different abstraction levels of process-oriented systems. IT-support plays a central role here, e.g. to assemble models [13], enable the reuse of best practice IT-processes [14] or manage extensions [15]. However, as in the reference-based model construction area, approaches offering recommendations are rarely implemented since the attention is more geared towards reusing configuration knowledge and IT-assets attached to process definitions.

Query-based support for model construction. Another field of research is concerned with finding models or model fragments which can be used to complete the model under construction. Koschmider [16] describes an approach and a tool to determine the similarity of the model currently under construction compared to models which already exist in a repository. In this 
approach, queries are generated "behind the scenes" in the tool which automatically translates the model under construction to a query for the repository. The described approach as well as other approaches based on query languages are invoked manually and tend to offer more coarsegrained modeling support such as lists of ranked similar models.

Pattern-based model construction. Patterns originate from the discipline of architecture and have been introduced as analysis patterns in conceptual modeling by Fowler [17]. They represent knowledge in the form of abstract templates used for applying well-known solutions to similar problems. Up to now, research has mainly focused on pattern detection (e.g. [18], [19]), although a few approaches support modeling [20]. In comparison to other construction support techniques, patterns are more abstract in nature and usually require a thorough interpretation by the modeler, in contrast to element suggestions implemented by recommender systems.

\subsection{On Recommender Systems}

Since the early 1990s, recommender systems have been used to recommend items such as news group messages, videos or music to users based on their individual taste or preferences. Videos and electronic commerce products are still the primary type of recommended items in the recommender systems research community due to the availability of huge data sets used for user profile analysis [21]. In practice, the application of recommender systems extends well beyond these traditional areas and nowadays also encompasses domains such as travelling, accommodation or social networks. Predominant recommendation paradigms are collaborative filtering, content-based filtering, knowledge-based recommendation, and hybrid approaches [21, p. 81]. Whereas collaborative filtering means that recommendations are based on the preferences of similar users, content-based recommendations are based on the properties of items. Knowledge-based approaches require some extra codified knowledge such as a product taxonomy in conjunction with recommendation rules.

In general, there is a plethora of approaches for combining these paradigms and calculating recommendations. There is no single superior approach for achieving best recommendations in all use cases. Rather, the quality and usefulness of the recommendations is highly dependent on the respective domain. It thus has to be evaluated for each use case separately. A further characteristic of recommender systems is that complex systems do not always perform better in evaluations than simple systems. Combining several recommendation paradigms and performing complex (and computationally expensive) calculations may not automatically lead to better recommendations than, e.g. simple top-k rating recommendations (see, e.g. Jannach et al. [22, p. 207]). Consequently, an incremental research approach in which, initially, a system is built which later is improved according to the feedback, seems to be an adequate way to explore the use of recommender systems in business process modeling. We follow also such a research approach. Therefore, we have implemented a recommendation tool combing item- and knowledge-based recommending augmented with implicit user rating. Whereas in previous publications we reported on the requirements [4] and generic data model [23], [24], for process modeling recommender systems, we in this publication explore user expectations and contrast them with first empirical findings.

\subsection{Recommender Functions in the SEMPHIS-Project}

Models of business processes are more important than ever as multiple objectives can be achieved by them: the documentation of the business processes, the optimization of these processes as well as the development, customization and integration of the involved IT systems. But process models are not easy to create. Especially for novice modelers, it is often difficult to maintain a uniform level of abstraction and to detect the relevant procedures without gaps. Therefore, in SEMPHIS research project, an adaptive and intelligent modeling tool is developed, which simplifies and accelerates business process modeling via predefined semantic building 
blocks and patterns, which are offered to the user through an intuitive and easy to use assistant function. Specifically, we support the modeler in deciding which activity should be inserted next in the process model. This functionality has been implemented in two preliminary prototypes.

Firstly, a traditional modeling tool has been extended with modeling assistant allowing to refine model elements with more detailed information (see Figure 1) [25]. To do this, the user can right-click on elements marked with an info-symbol, retrieve the detailed information and can insert this information automatically into the model.

However, the detailed information is fixed and can only slightly be modified, e.g. by omitting elements from insertion. Therefore, we plan to design and implement a more advanced recommender functionality that offers a ranked list of model element suggestions. Thus, a prototype of this recommender service is already implemented as a web-based prototype (see Figure 2). It offers the user suggestions which are ranked according to the industry (e.g. health or automotive), the process category (e.g. sales or production) and, in particular, with the frequency with which suggestions have actually been inserted into the model during past modeling activities of the user the aggregated ranking value is depicted (the bar chart on the right side of the screenshot shown in Figure 2).

Using the parameters described, which can be weighted according to the user preferences, it is possible to adapt the guidance in process modeling according to different requirements. This, in turn, calls for empirical investigation since the established parameters and their weights cannot be determined using existing theories of business process modeling. Therefore, we are gathering first feedback from potential users and experts in order to validate our assumptions and design choices.

\section{Research Process}

The research presented in this article followed a structured process that is illustrated in Figure 3. It included the feedback from fairgoers as well as key findings from an experiment.

In an initial phase (1), we prepared our prototype systems originating from the research project SEMPHIS and created a questionnaire for gathering the feedback. We, moreover, considered several options of how to perform the data collection such as sending the questionnaire or demonstrating the prototype followed by a survey. We finally opted for the latter strategy since, first, we aimed at presenting the interviewees our research prototypes personally in order to get a richer and more immediate feedback. Such a presentation would not be possible, when sending the questionnaires, e.g. via e-mail. In addition, after our presentation, we wanted to give the interviewees also the chance to try our tools out. Secondly, convenience sampling - as performed in this study - is especially suited for preliminary studies concerning, e.g. pilot testing [26]. It belongs to the group of non-probability sampling and is a sampling technique where the samples are gathered in a process that does not give all the individuals in the population equal chances of being selected [27]. Therefore, it is also considered to be not generalizable [26]. Nonetheless, it is useful for getting first feedback at an early stage. The questionnaire itself was organized in three parts: (a) personal information about the interviewees, (b) feedback on the functionalities presented, and (c) gathering additional requirements that seem to be useful for being additionally implemented.

In the next phase (2), we presented our prototypes at the world's leading high-tech event CeBIT (www.cebit.de) in Hannover, Germany, where interested fairgoers where recruited and filled-out our feedback form. In phase (3), feedback was analyzed and fairgoers expectations towards business process modeling recommender systems were elicited (cf. Section 4). The results were finally, in phase (4), confronted with the results from an experiment (cf. Section 5) leading to final overall findings (cf. Sections 5 and 6). 


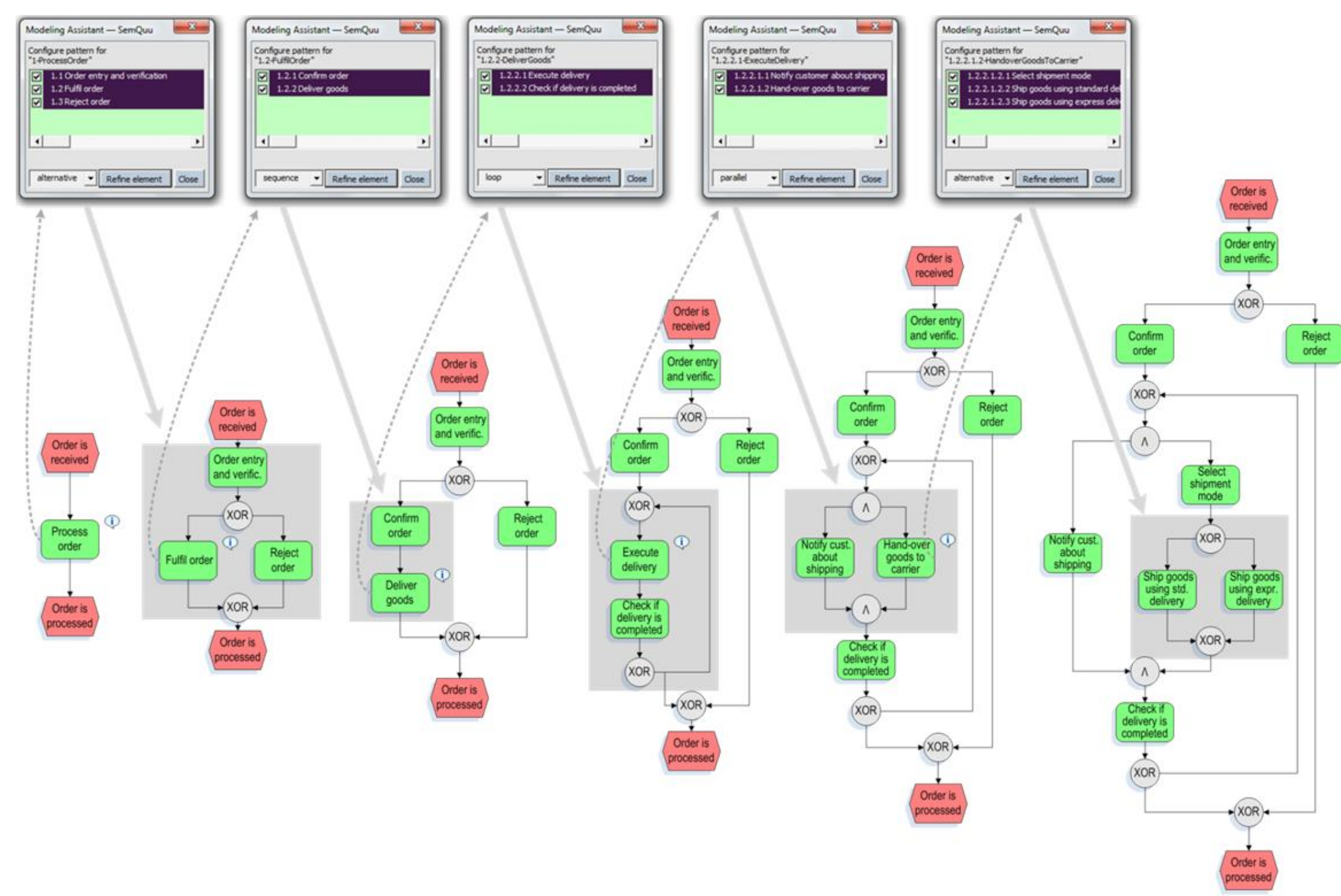

Figure 1. Extension of a modeling tool (Microsoft Visio) with a modeling assistant feature 

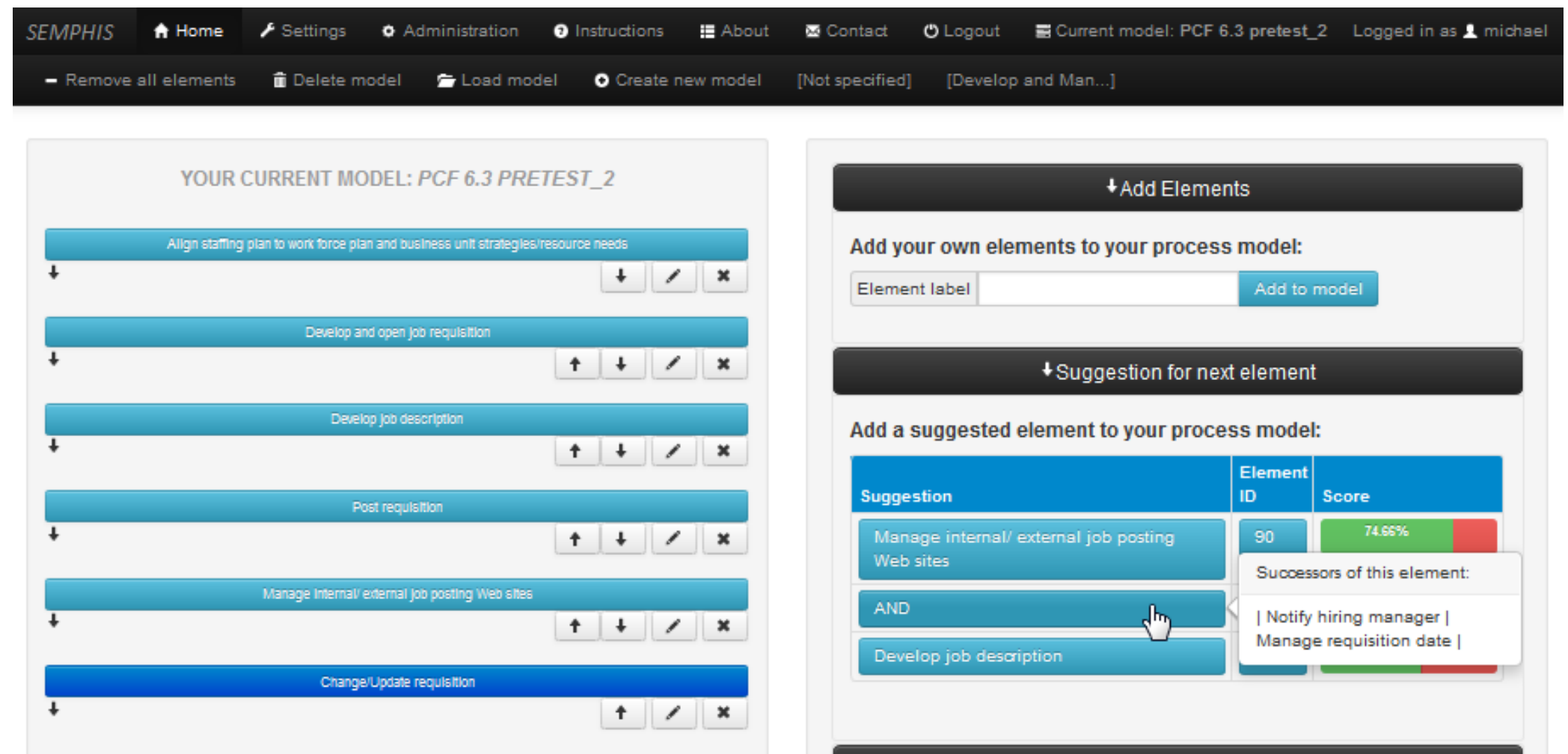

Add a suggested element to your process model:

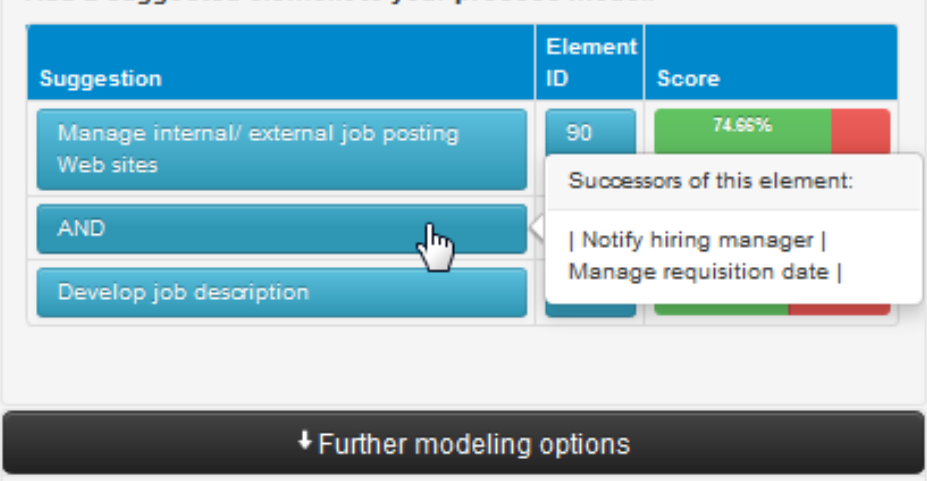

Create SplitJoin-Operator

Figure 2. Web-based recommender demo for business process modeling 


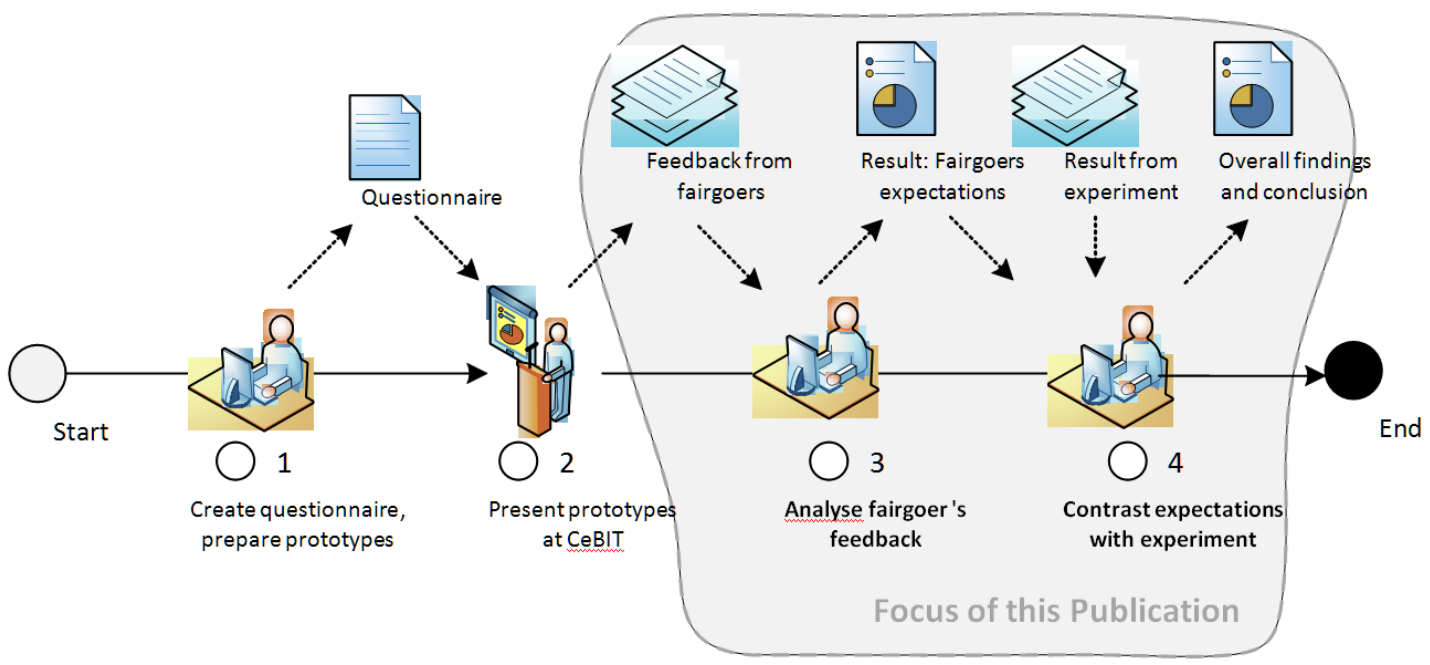

Figure 3. Research process

\section{Feedback from Fairgoers}

\subsection{Information About the Interviewees}

The first part of our survey dealt - as mentioned earlier - with personal information about the interviewees (see Figure 4). The first goal of this part was to get an overview of the people that participated. The second goal was to eventually identify correlations with respect to the other parts of the survey by calculating correlation coefficients. We were able to attract 78 visitors in total and to present them our prototypical implementations. All 78 visitors have been asked to fill out our survey, of which 66 were willing to do so. Since the survey was the last step after our presentation, visitors not interested in process modeling already escaped our presentation before we could ask them to fill out the survey. Therefore, all visitors that finally filled out the survey had at least moderate knowledge on process management or process modeling.

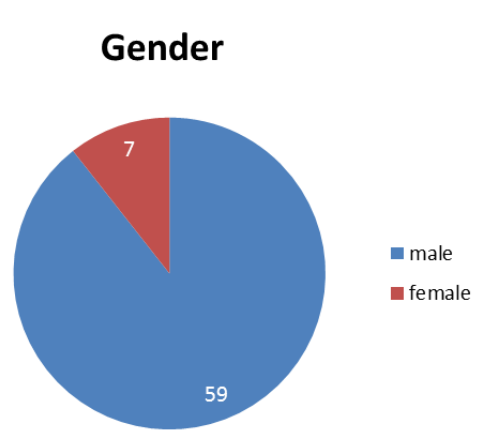

(a)

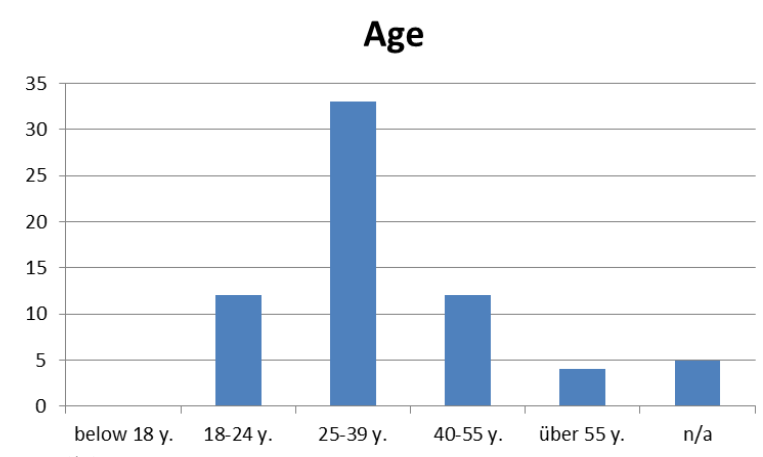

(b)

Figure 4. Gender and age of the respondents

As can be seen from Figure 4a, most of the respondents that were interested in the presentation of our prototypes were male visitors. This can probably be explained by the fact that we were presenting our exponate at a technology-intensive fair, which had also in past years a much higher percentage of male visitors ${ }^{\dagger}$. It is also interesting to have a look at the age of the

\footnotetext{
${ }^{\dagger}$ For instance, $90 \%$ of the fairgoers at CeBIT 2002 were male: http://www.zdnet.de/2106960/cebit-18-prozent-wenigermessebesucher-in-2002/ (accessed on 14.02.2018)
} 
respondents. Figure $4 \mathrm{~b}$ depicts that most of respondents were between 25 and 39 years of age. The two categories 40-55 years and 18-24 years were represented equally by 12 respondents each. One can conclude from this data that most respondents had a relevant working age, including with the latter category also people that are up to finish their studies and to start their working career. This brings us to the following two criteria that have been analyzed, namely the educational background and the working experience (see Figure 5).

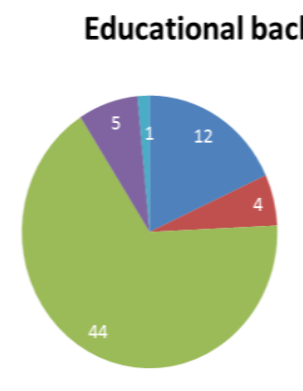

(a)

\section{Experience}

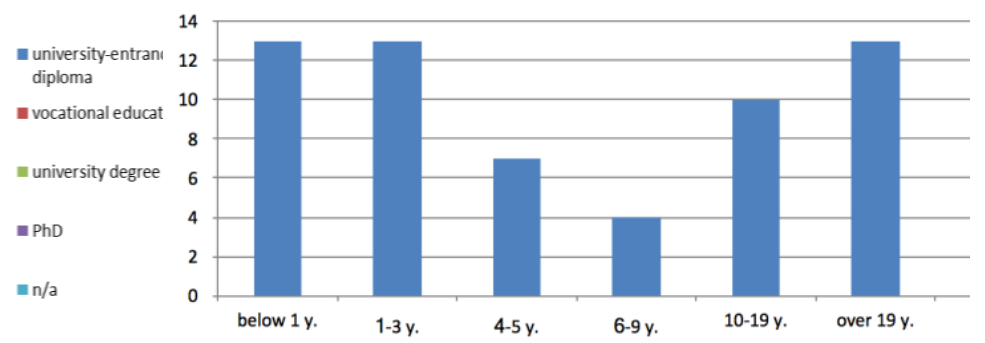

(b)

Figure 5. Educational background and working experience of the respondents

It can be seen from Figure 5a that a high majority of respondents had a university degree (44 respondents), accompanied by five PhDs. A university-entrance diploma was possessed by 12 respondents, which exactly corresponds to the category 18-24 years from Figure $4 \mathrm{~b}$. Additionally only a small number of respondents (four people) had no university education, but possessed a vocational education. This information is quite relevant, because it shows that the high majority of respondents were highly educated and probably had specific skills. By moving to the working experience expressed in years we can observe that 23 respondents had more than 4 years of working experience. However, we can see a similar number of people that had working experience of less than three years. Below, we present two further criteria that were analyzed in the first part of our survey: the type of industry and the size of the organization/company where the respondents were affiliated.

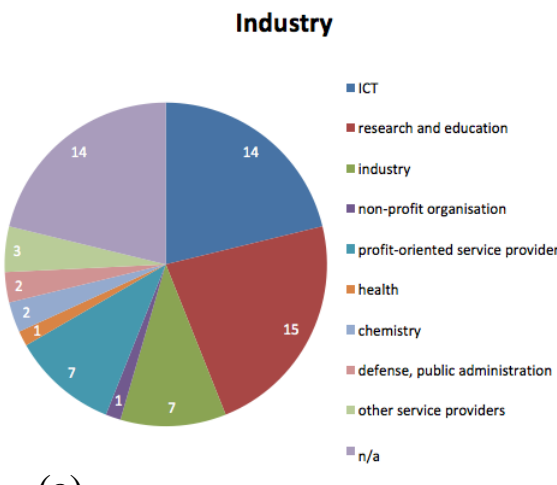

(a)

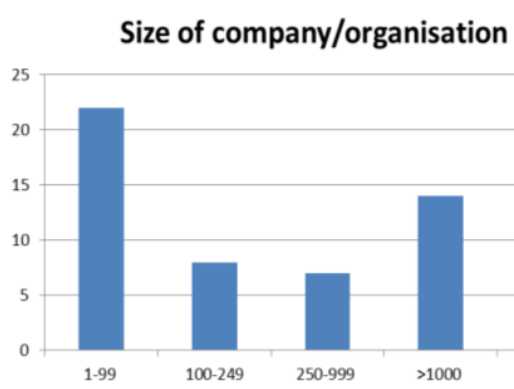

(b)

Figure 6. Industry type and company/organization size of the respondents

The most represented types of industry were information and communication technology (ICT) and research and education. There was also a big category that did not fit into one of the above industry types, as can be seen on the very right of Figure 6a. The size of company/organization showed that the largest category was made up of so-called small and medium sized enterprises (SMEs). The rest of the respondents were affiliated to bigger companies and organizations. 


\subsection{Perception of Current Prototypes}

The second part of our survey was centred on the second point in the research process (see Figure 3), where we have shown short demonstration of the functionalities that our prototypes offer. Our main intention was to collect first feedback from fairgoers. The overall judgment of the interviewees tackled five points, namely to rate (a) how innovative they assess the prototypes, (b) whether reduction of complexity in the modeling process could be achieved, (c) whether our approach would have an impact on the speed of modeling of business processes, (d) how complete the created process models would be indeed, and finally (e) whether the application of our approach would lead to the development of creative solutions. For getting answers to these points we applied a four-level Likert-like scale. A value of ++ is used to represent complete agreement whilst a value of -- is used to represent complete disagreement with the above mentioned points.

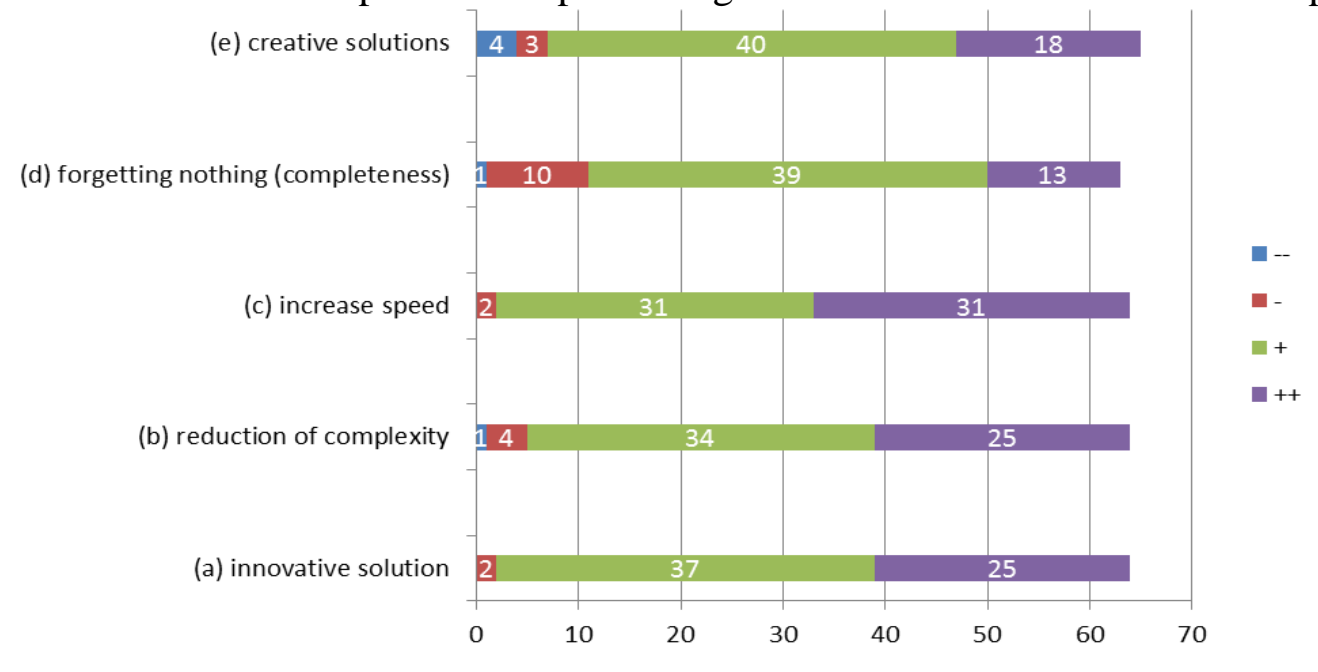

Figure 7. Expected benefit of using recommender features

Figure 7 shows the results of the second part of our survey at a glance. It can be seen that all points were evaluated from a vast majority in a positive way. This provides us a first justification of our work. In particular the respondents had the highest consent on the increased speed (c), i.e. a faster creation of business process models, where only two respondents disagreed. As can be seen, a similar high affirmation holds for (e) the creation of innovative solutions, (b) the reduction of complexity while performing modeling tasks, and (a) the creation of innovative solutions. The highest number of disagreements can be observed with respect to (d) the completeness of the resulting business process models, where we have a two-digit number of disagreements. Many fairgoers stated that the completeness of the created models depends on the lexical knowledge in form of semantic building blocks and not on the prototypical solution itself. Additionally, to the analysis shown in Figure 7, we also calculated correlations between the personal information of the respondents and the results of this subsection. The correlations are depicted in Table 1.

In addition to our survey, we also have documented conversation contents. One important comment - raised by multiple fairgoers - was concerned with the applicability of the approach in different industries, because each industry has next to generally existent processes also its industry-specific processes. 
Table 1. Correlations between the first and the second parts of survey responses

\begin{tabular}{|l|l|}
\hline Correlation 1 & $\begin{array}{l}\text { Male respondents thought that the solution is innovative. } \\
\text { (Correlation coefficient: } 0.249^{*} \text { ) }\end{array}$ \\
\hline Correlation 2 & $\begin{array}{l}\text { Female respondents thought that the solution is not innovative. } \\
\text { (Correlation coefficient: }-0.249^{*} \text { ) }\end{array}$ \\
\hline Correlation 3 & $\begin{array}{l}\text { The respondents feel with increasing age and hence normally with } \\
\text { increasing work experience that the solutions will not contribute to } \\
\text { the reduction of complexity. } \\
\text { (Correlation coefficient: -0.210*) }\end{array}$ \\
\hline Correlation 4 & $\begin{array}{l}\text { Respondents affiliated to companies/organizations from 100-249 } \\
\text { employees think that the resulting solutions are innovative. } \\
\text { (Correlation coefficient: } 0.309^{*} \text { ) }\end{array}$ \\
\hline Correlation 5 & $\begin{array}{l}\text { Respondents affiliated to companies/organizations with more than } \\
\text { 1000 employees think that the resulting solutions are not } \\
\text { innovative. } \\
\text { (Correlation coefficient: -0.236*) }\end{array}$ \\
\hline *The correlation is significant at 0.05 levels (two-tailed).
\end{tabular}

\subsection{Key Findings from Fairgoer-Feedback}

The most important finding results from the judgment about the expected benefit of using recommender features (cf. Figure 7). When looking back to Figure 7, we notice that the statement with the highest number of participants that either fully or partially support it (i.e. rated $++/+)$ is "increase speed". So far, this is quite intuitive since inserting modeling suggestions (provided that they are relevant) saves time since the modeler is not required to type in the model element labels. More interesting is the statement with the highest number of participants that either do not at all agree or only partially agree (i.e. rated --/-). This statement is "forgetting nothing (completeness)". Before the experiment, we thought that creating more complete models and not forgetting important steps while modeling would be among the top benefits or even the top benefit of a process modeling recommender system. To our great surprise, completeness of the model is the least supported benefit, i.e. the benefit that participants consider the most unlikely to occur.

Even though the results are representative for the group of fairgoers only (due to so called convenience sampling, cf. Section 3), they nevertheless give first useful insights by potential users. Due to the counterintuitive nature of the results we contrast the results of the fairgoer feedback analysis with the results of an experiment we conducted in the same year. The experiment addresses (amongst others) the question of modeling speed and modeling completeness. For this reason, the experiment is suitable to contrast the results described in this section.

\section{Results from an Experiment}

\subsection{Introduction to the Experiment}

The goal of the experiment that has been performed was to get a first impression of how assistance-based modeling could influence modeling tasks, most notably the labeling of process model elements. The experiment is described shortly in [28]. However, in this article, we provide a more detailed description, show the user interface of the prototype we used in the experiment and provide an in-depth interpretation of the results. We also later contrast the core findings of the experiment with the empirical results and insights we obtained from the fairgoers. The core of the experiment was to provide modelers with process knowledge offered by a tool and to observe the impact on modeling results. For this purpose, several modeling tasks were formulated which participants had to solve. While doing so, a prototypical tool could be used by 
the participants to retrieve suggestions for modeling and get inspired about the content of process models. The suggestions were computed using an ontology-based version of the MIT Process Handbook. MIT Process Handbook is a large collection of process knowledge covering approximately 8000 business processes. It is online accessible (process.mit.edu/) and has been released in an ontology-based version encoded in the Web Ontology Language (OWL) under an Apache 2.0 license from the "Dynamic and Distributed Information Systems Group" (DDIS) around 2006. Access to this knowledge was made available using a browser interface which had been implemented for the experiment (see Figure 8). The interface mainly enabled the participants to search and browse the MIT Process Handbook library.

\begin{tabular}{|c|c|c|c|}
\hline \multicolumn{2}{|c|}{ The Process Handbook } & \multicolumn{2}{|l|}{+} \\
\hline \multicolumn{4}{|c|}{ Search the Process Handbook for a process matching your keywords: } \\
\hline \multicolumn{4}{|l|}{ sell } \\
\hline \multicolumn{4}{|c|}{ Number of results: $10 \quad$} \\
\hline \multicolumn{4}{|c|}{ Search Elements: process $\quad$} \\
\hline \multicolumn{4}{|c|}{ Search Tags: name $\quad$} \\
\hline \multicolumn{4}{|c|}{ search } \\
\hline No. & ID & Process & Short Description \\
\hline 1 & $\underline{\underline{E} 02639}$ & Sell & $\begin{array}{l}\text { Selling implies an exchange of value from the customer to the seller for a product and/or service. } \\
\text { Note that the subactivities in }\end{array}$ \\
\hline 2 & E07982 & Sell service & This activity is the simple case of selling a service. \\
\hline 3 & $\underline{E 08023}$ & Sell product & $\begin{array}{l}\text { This activity is the general case of selling a product. The only difference between selling a product } \\
\text { and service at this level of }\end{array}$ \\
\hline 4 & $\underline{E 14556}$ & Sell direct & [] \\
\hline 5 & $\underline{E 14564}$ & $\begin{array}{l}\text { Sell to } \\
\text { consumers }\end{array}$ & $\begin{array}{l}\text { Selling implies an exchange of value from the customer to the seller for a product and/or service. } \\
\text { Note that the subactivities in }\end{array}$ \\
\hline 6 & $\underline{E 14566}$ & $\begin{array}{l}\text { Sell to } \\
\text { businesses }\end{array}$ & $\begin{array}{l}\text { Selling implies an exchange of value from the customer to the seller for a product and/or service. } \\
\text { Note that the subactivities in }\end{array}$ \\
\hline
\end{tabular}

Figure 8. Browser-based interface for searching relevant processes

In order to search for process knowledge, exact phrases could be typed in as well as keywords separated by semicolons ";". Amongst other parameters, most notably the maximum number of results to be displayed could be restricted. Once a search is executed, a list of processes appears with ID, short name and description. If a process is selected, it is possible to obtain further information on the process. For instance, a full description is displayed. Additional navigation links are also provided to retrieve and explore the following process knowledge: (i) parts of the process, i.e. individual steps in processes, (ii) the parent-process where this process is a part of, (ii) specializations of the process, e.g. for specific types of industries or to reflect alternative methods of conducting the process; as well as (iv) generalizations of the process, which is the opposite direction of specializations; Figure 9 provides an overview of the available information with the example of the coarse-grained process "Design product and process". As it can be seen from Figure 9, especially the part-of-information reveals useful hints about what could be included in the process model.

During the experiment, all six participants first received small textual instructions (approx. half a page) reflecting the desired contents of the process models. The topics of the processes were (i) hiring an employee, (ii) sales management, and (iii) creation and analysis of advertisements. After reading the instructions, all participants had to create, in sum, six models that describe the processes. The focus of modeling was to specify activities in a meaningful order. Providing control flow other than a sequence flow as well as exception management were 
not mandatory. For each topic, one model had to be created with the help of the process library and another without the help. The experiment took place at a laboratory of Osnabrück University and most participating modelers were post-graduate students at Master or PhD levels. After the experiment, the participants were interviewed in an in-depth interviews with no predefined structure in order to additionally capture participants' ideas and feelings about the assistance system.

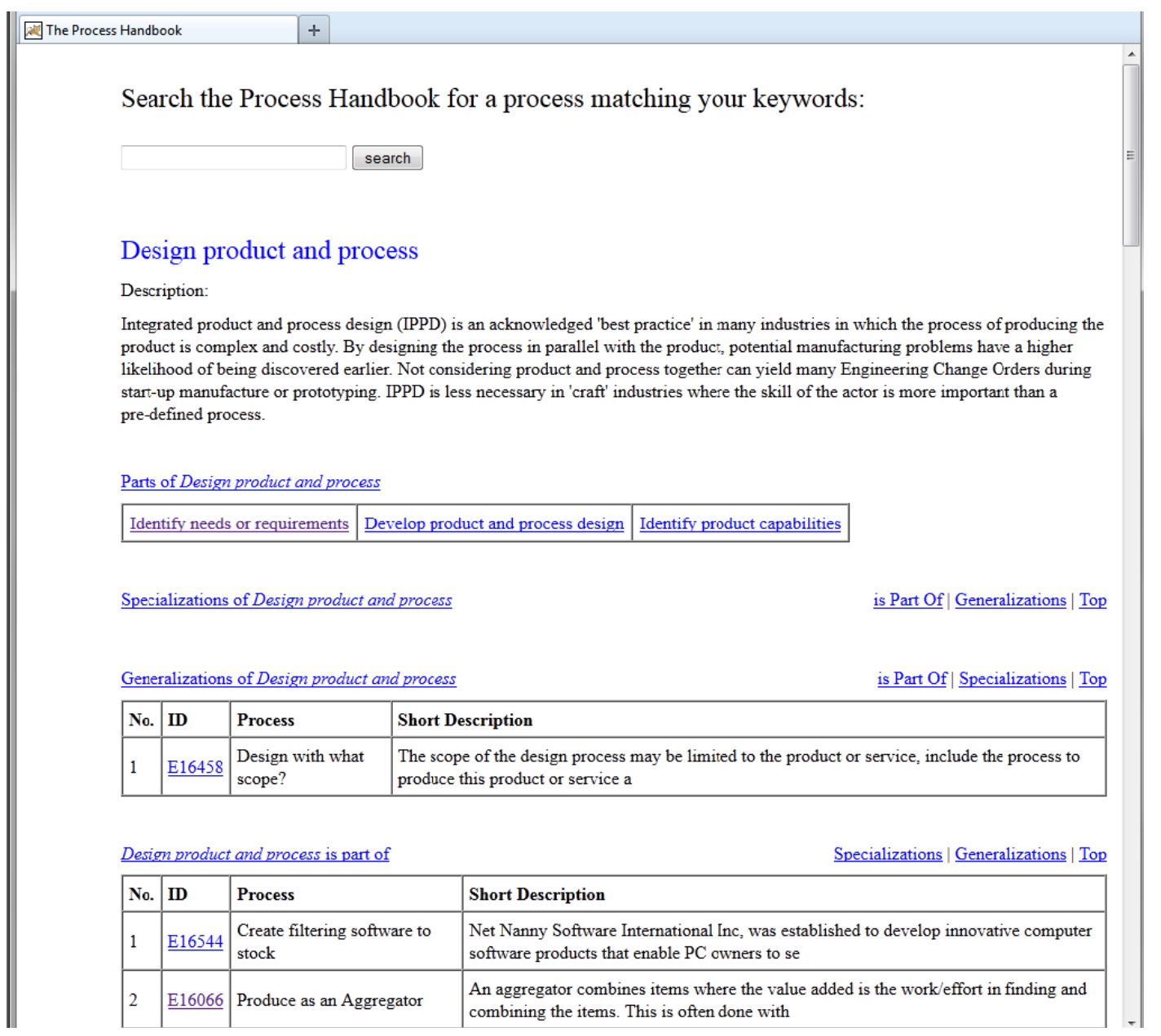

Figure 9. Detailed information and navigation instruments of the prototype

\subsection{Key Findings of the Experiment}

The assessment of the results centered on two aspects: The number of added elements due to using the process library and time consumption. Regarding the first aspect, Figure 10 shows the number of additionally inserted model elements by assisted modeling. Bars that belong to one participant are grouped on the $x$-axis and labeled with 1,2,3 according to the model topic (see previous section), while on the $y$-axis the number of model elements is shown. For 4 participants out of 6 , overall the modeling process had benefitted from knowledge-support by the process library. It does so in the way that elements have been added to the models (orange color), which were beforehand absent while modeling without the support (dark blue color). The amount of additionally inserted model elements ranges from plus $16 \%$ to $60 \%$ thus demonstrating that there is a positive effect in regard to the number of model elements. 


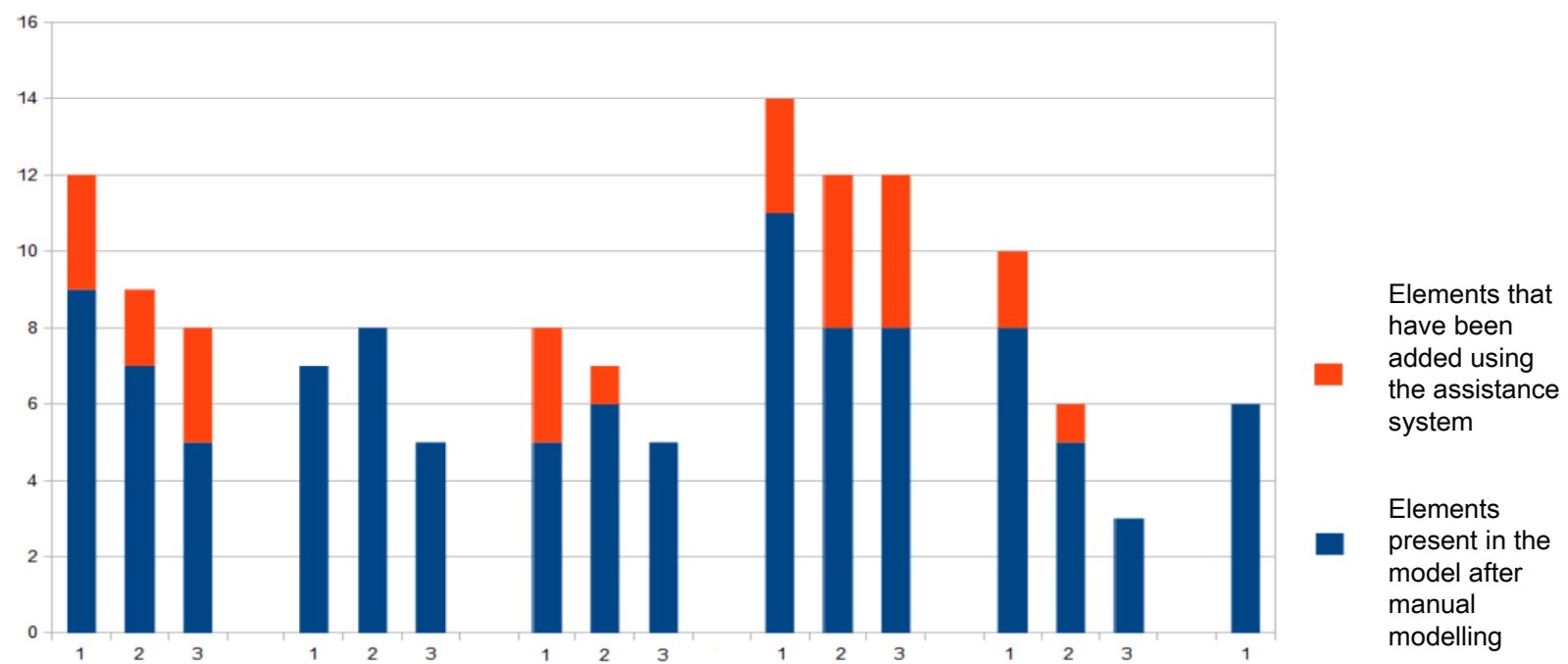

Figure 10. Additionally inserted model elements by assisted modeling

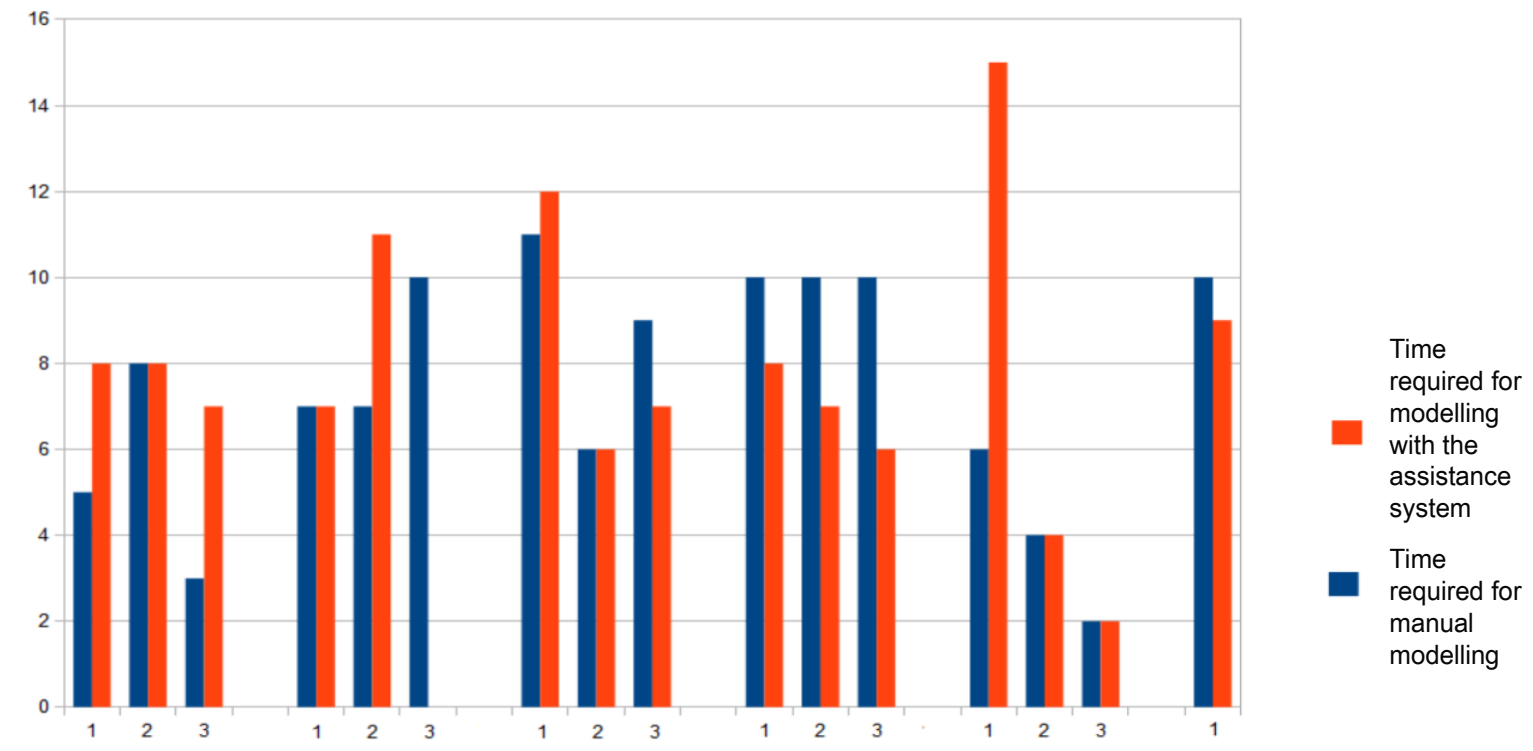

Figure 11. Assessing the time needed to create models with assistance and without assistance

By manually inspecting the additional elements in the process model, it turned out that these elements were meaningful and supported well the modeling goal. This has also been confirmed by various statements in the interviews. For instance, a participant made the following statement: "If there had been suggestions, they helped me to focus on the right abstraction level and on what's really important. It somehow increased my confidence in what I am modeling and therefore I liked it very much."

The second criterion was to determine also differences in the time consumption needed to create a process model. Here, the picture is quite less clear (cf. Figure 11). Only in 5 cases, time consumption was decreased when modeling with the help of the process library in contrast to modeling without the help. It stayed the same for 5 models and time consumption was even increased for 5 models with the help of the process library in comparison to modeling without the help.

\subsection{Contrasting the Expectations with the Experiment Results}

Surprisingly, the judgments of the fairgoers in regard to model completeness and modeling speed could not be confirmed by the experiment. Even given the fact that insights from the experiment 
have to be drawn with a reasonable amount of caution due to the low number of participants, it comes nonetheless to our surprise that the results from the experiment seem to point into exactly the opposite direction from what fairgoers think.

Regarding completeness of models, a cause for this contradiction might be that fairgoers intuitively thought of process modeling as a rather simple mapping of as-is processes in their enterprise or organization. From this perspective, it is quite likely that modeling suggestions generated from a tool will not cover exactly what the modeler needs to express - hence that they do not contribute to completeness. However, in the experiment it was demonstrated that modelers confronted with the task of creating a process model out of a rather short and vague text in fact leveraged external process knowledge and made their models more encompassing or complete - and appreciated this approach. What can be concluded from this contradiction is that the process modeling support, in order to be useful, should either precisely cover the domain that is to be modeled or, instead, be applied to inspire modeling in a scenario permitting modeling freedom, i.e. when modeling is not entirely about mapping an existing as-is process.

Regarding the mismatch concerning time consumption for modeling activities, a majority of fairgoers expected an increase of modeling speed. This is in contrast to the measured modeling speed in the experiment that showed a partial slowing down of the modeling process. An explanation for the decrease in modeling speed is that the processes and their process parts listed in the MIT Process Handbook were fairly abstract. So, the modeler had to think about these processes and whether and how they could be adapted and inserted in the currently modeled process. What can be concluded from this mismatch is that time consumption will increase when process contents are not provided on the right level of detail or abstraction. Hence, in order to support the user efficiently, process knowledge should be provided at the right level of granularity.

Summarizing these preliminary conclusions that have yet to be confirmed conducting future research, we can hypothesize that two "sweet spots" of assisted process modeling exist:

- Support of fast configuration-oriented modeling. The modeler mainly re-arranges preconfigured knowledge. This knowledge is represented inside the modeling assistance tool and captures precisely the domain or process that is to be modeled. Modeling suggestions are on the right level of granularity. In this scenario, the assistance system permanently suggests the next matching element and removes the burden to label model elements. The modeler hence saves time and effort. It suits best in situations where existing processes have to be reconfigured or re-ordered, e.g. when a new production process should be implemented based on the composition of existing and known capabilities.

- Support of modeling as problem solving. The modeler is confronted with a difficult modeling task. Requirements are unclear as well as the process border and required level of granularity. Knowledge represented inside the modeling assistance tool provides orientation in capturing broad knowledge about the domain that is to be modeled on different levels of detail. Modeling suggestions are meant to inform the modeler about typical tasks as well as alternatives. In this scenario, the modeler gets inspired by the assistance system, thinks about suggestions and possibly adapts some suggested elements to fit his or her purposes before adding them to the model. It suits best when efficiency in terms of time consumption is not the primary goal, but instead, the goal is to successfully solve a challenging process design problem.

\section{Conclusion}

Our preliminary analysis of recommender functionalities applied to business process modeling shows interesting results. First of all, we analyzed feedback collected from fairgoers. We presented them recommender tools and they had the opportunity to test the tool in reality. After this, a survey was filled out. Important results from this analysis are that fairgoers expected an increased modeling speed but were sceptical about the contribution of modeling assistance 
regarding model completeness. This finding was contrasted with the results of an experiment where the results pointed in the opposite direction. Modelers, in fact, appreciated process modeling assistance and included additional model elements into their models. However, a general increase in modeling speed could not be reported. These mismatches then led to some preliminary conclusions that have yet to be confirmed by future research. The essence of these conclusions is the hypothesis that two "sweet spots" of assisted modeling might exist: first, fast configuration-oriented modeling where the modeler mainly re-arranges pre-configured knowledge represented inside a tool; and, second, modeling as problem solving where the modeler seeks inspiration, thinks about and adapts concepts from a reference work.

Future research is needed to gain more insights into the effects that process modeling recommender systems exert both on the modeling process and on the modeling result. Closely connected to that, also more research is needed towards finding the "sweet spots" of assisted modeling. The initial considerations given in this contribution might serve as a starting point for this.

\section{References}

[1] P. Melville and V. Sindhwani, "Recommender Systems," in Encyclopedia of Machine Learning, Springer, 2010.

[2] F. Ricci, L. Rokach, B. Shapira, and P. B. Kantor, Recommender Systems Handbook, Springer, 2011. Available: https://doi.org/10.1007/978-0-387-85820-3

[3] S. Moore, Gartner Says Spending on Business Process Management Suites to Reach \$2.7 Billion in 2015 as Organizations Digitalize Processes. Gartner Newsroom Press Release, 2015.

[4] M. Fellmann, N. Zarvic, D. Metzger, and A. Koschmider, "Requirements Catalog for Business Process Modeling Recommender Systems," in Proceedings of Wirtschaftsinformatik (WI 2015), Osnabrück, Germany, 2015.

[5] K. Sarshar, M. Weber, and P. Loos, "Einsatz der Informationsmodellierung bei der Einführung betrieblicher Standardsoftware : Eine empirische Untersuchung bei Energieversorgerunternehmen," Wirtschaftsinformatik, vol. 48(2), pp. 120-127, 2006. Available: https://doi.org/10.1007/s11576-006-0026-X

[6] A. Nielen, D. Költer, S. Mütze-Niewöhner, J. Karla, and C. Schlick, "An Empirical Analysis of Human Performance and Error in Process Model Development," in Conceptual Modeling (ER 2011), Lecture Notes in Computer Science, vol. 6998, pp. 514-523, 2011. Available: https://doi.org/10.1007/978-3-642-24606-7_42

[7] I. Wilmont, S. Brinkkemper, I Weerd, and S. Hoppenbrouwers, "Exploring Intuitive Modelling Behaviour," in Enterprise, Business-Process and Information Systems Modeling, Lecture Notes in Business Information Processing, vol. 50, Springer, pp. 301-313, 2010. Available: https://doi.org/10.1007/978-3-642-13051-9_25

[8] M. Fellmann, N. Zarvic, and O. Thomas, "Business Processes Modelling Assistance by Recommender Functionalities: A First Evaluation from Potential Users," in Proceedings of Business Information Research Conference (BIR 2017), Lecture Notes in Business Information Processing, vol. 295 Springer, pp. 79-92, 2017. Available: https://doi.org/10.1007/978-3-319-64930-6_6

[9] P. Fettke and P. Loos, "Methoden zur Wiederverwendung von Referenz modellen - Übersicht und Taxonomie," in Arbeitsberichte Nr. 90, Institut für Wirtschaftsinformatik, Westfälische Wilhelms-Universität Münster, 2002.

[10] M. Remme, Konstruktion von Geschäftsprozessen: Ein modellgestützter Ansatz durch Montage generischer Prozeßpartikel, Gabler (Schriften zur EDV-orientierten Betriebswirtschaft), Wiesbaden, 1997.

[11]U. Frank, S. Strecker, and S. Koch, ““'Open Model” - ein Vorschlag für ein Forschungsprogramm der Wirtschaftsinformatik," in Proc. of 8. Int.1 Tagung Wirtschaftsinformatik (WI 2007), Karlsruhe, Germany, vol. 2., pp. 217-234, 2007.

[12]D. Kopperger and J. Drawehn, "Qualitätsmanagement, Referenz-und Reifegradmodelle,” in BPM Tools 2008 Eine evaluierende Marktstudie zu aktuellen Werkzeugen, Fraunhofer-Verlag, Stuttgart, pp. 71-91, 2008.

[13] S. Zlatkin and R. Kaschek, "Towards Amplifying Business Process Reuse," in Perspectives in Conceptual Modeling : Proc. of the 6th Int.l Workshop on Conceptual Modeling Approaches for e-Business (eCOMO 2005), Lecture Notes in Computer Science, vol. 3770, Springer, pp. 364-374, 2005. Available: https://doi.org/10.1007/11568346_39 
[14]H. R. Motahari-Nezhad, S. Graupner, and C. Bartolini, “A Framework for Modeling and Enabling Reuse of Best Practice IT Processes," in Business Process Management Workshops, BPM 2010, Lecture Notes in Business Information Processing, vol. 66, Springer, pp. 226-231, 2011. Available: https://doi.org/10.1007/9783-642-20511-8_22

[15] S. Balko, A. H. M. Ter Hofstede, A. P. Barros, M. La Rosa, and M. J. Adams, "Business process extensibility," Enterprise Modelling and Information Systems A.rchitectures Journal, vol. 5, no. 3, pp. 4-23, 2010.

[16]A. Koschmider, “Ähnlichke,itsbasierte Modellierungssunterstützung für Geschäftsprozesse,” Dissertation an der Universität Karlsruhe, Fakultät für Wirtschaftswissenschaften, 2007.

[17] M. Fowler, Analysis Patterns: Reusable Object Models, Addison-Wesley Longman, Amsterdam, 1996.

[18] J. M. Lau, C. Iochpe, L. H. Thom, and M. Reichert, "Discovery and analysis of activity pattern co-occurrences in business process models," in Proc. of the 11th Int.1 Conf. on Enterprise Information Systems (ICEIS 2009), pp. 83-88, 2009. Available: https://doi.org/10.5220/0001958800830088

[19] P. Delfmann, S. Herwig, L. Lis, and A. Stein, "Pattern Matching in Conceptual Models - A Formal MultiModelling Language Approach," in Proc. of the 3rd Int.l Workshop Enterprise Modelling and Information Systems Architectures (EMISA 2009), pp. 13-27, 2009.

[20] L. H. Thom, C. Iochpe, M. Reichert, B. Weber, D. Matthias, G. S. Nascimento, and C. M. Chiao, "On the Support of Activity Patterns in ProWAP: Case Studies, Formal Semantics, Tool Support," Revista Brasileira de Sistemas de Informação (iSys), vol. 1, no. 1, pp. 27-53, 2008

[21]D. Jannach, M. Zanker, M. Ge, and M. Gröning, "Recommender Systems in Computer Science and Information Systems - A Landscape of Research," in E-Commerce and Web Technologies, EC-Web 2012, Lecture Notes in Business Information Processing, vol. 123, Springer, pp. 76-87, 2012.

[22] D. Jannach, M. Zanker, A. Felfernig, and G. Friedrich, Recommender Systems - An Introduction, Cambridge University Press, 2011.

[23] M. Fellmann, D. Metzger, and O. Thomas, "Data Model Development for Process Modeling Recommender Systems," in The Practice of Enterprise Modeling, PoEM 2016, Lecture Notes in Business Information Processing, vol. 267, Springer, pp. 87-101, 2016. Available: https://doi.org/10.1007/978-3-319-48393-1_7

[24] M. Fellmann, D. Metzger, S. Jannaber, N. Zarvic, and O. Thomas, "Process Modeling Recommender Systems," Business Information System Engineering, vol. 60(1), pp. 21-38, 2018. Available: https://doi.org/10.1007/s12599-018-0517-5

[25] M. Fellmann and O. Thomas, "Process Model Verification with SemQuu," in Enterprise Modelling and Information Systems Architectures (EMISA 2011), pp. 231-236, 2011.

[26] I. Boxill, C.-M. Chambers, and E. Wint, Introduction to Social Research: With Applications to the Caribbean, Canoe Press, University of the West Indies, 1997.

[27] B. J. Oates, Researching Information Systems and Computing, SAGE Publications, 2006.

[28] M. Fellmann, N. Zarvic, A. Sudau, and L. Nobbe, “Ontology-Based Assistance for Semi-Formal Process Modeling," in Proc. of the 5th International Workshop on Enterprise Modelling and Information Systems Architectures (EMISA 2013), pp. 119-132, 2013. 